\title{
Syndrome de fuite capillaire systémique après l'administration du vaccin ChAdOx1 nCOV-19 (Oxford-AstraZeneca)
}

\author{
Julie Robichaud MD PhD, Catherine Côté BPharm MSc, Fanny Côté MD
}

Citation : CMAJ 2021 August 30;193:E1341-4. doi : 10.1503/cmaj.211212-f; diffusion hâtive le 6 août 2021

Voir la version anglaise de l'article ici : www.cmaj.ca/lookup/doi/10.1503/cmaj.211212

$\mathbf{U}$

$\mathrm{n}$ homme de 66 ans est venu consulter au service des urgences parce qu'il présentait un malaise généralisé, des nausées, une douleur thoracique et des étourdissements depuis la veille. À l'examen, sa température était de $36,5^{\circ} \mathrm{C}$, sa pression artérielle, de $112 / 78 \mathrm{~mm} \mathrm{Hg}$, sa fréquence cardiaque, de 112 battements/min et sa saturation en oxygène, de $96 \%$ à l'air ambiant. Sa fréquence respiratoire était normale. Il a indiqué ne pas avoir d'allergies, ne pas consommer de drogues ou d'alcool de façon inappropriée et ne pas prendre de médicaments ni de produits en vente libre. Il avait reçu sa première dose du vaccin ChAdOx1 nCOV-19 (Oxford-AstraZeneca) 2 jours plus tôt.

Les antécédents médicaux du patient comprenaient une gammapathie monoclonale à signification indéterminée (immunoglobuline $\mathrm{G} \kappa[$ [IgG K]), ainsi qu'un arrêt cardiaque en 2017. À l'époque, il avait présenté une faiblesse généralisée et un épisode de syncope. Comme son taux d'hémoglobine était élevé (210 g/L [plage normale 130-180 g/L]), on avait soupçonné une polyglobulie et réalisé une phlébotomie. Peu après, le patient était devenu hypotendu et avait présenté un arrêt cardiaque caractérisé par la présence d'une activité électrique sans pouls. Il avait été réanimé, s'était rétabli sans déficits importants et avait reçu son congé 5 semaines plus tard. Il avait obtenu un résultat positif au dépistage de l'influenza de type $A$, et on avait estimé que le choc était attribuable à l'infection virale.

À l'examen actuel, le patient présentait un taux d'hémoglobine considérablement élevé ( $224 \mathrm{~g} / \mathrm{L})$, une hypoalbuminémie ( $28 \mathrm{~g} / \mathrm{L}$ [plage normale $34-55 \mathrm{~g} / \mathrm{L}$ ]) et un haut taux de créatinine (133 $\mu \mathrm{mol} / \mathrm{L}$ [plage normale $62-115 \mu \mathrm{mol} / \mathrm{L}])$. Ses paramètres de coagulation, ses enzymes cardiaques et hépatiques et ses taux de protéine C-réactive et de procalcitonine étaient normaux. Ses résultats au dépistage du SRAS-CoV-2 et au dépistage élargi des virus respiratoires étaient négatifs. Enfin, aucune particularité n'a été constatée à la radiographie thoracique, à la tomodensitométrie abdominale, à l'électrocardiographie et à l'échographie ciblée en traumatologie (tableau 1 et tableau 2).

\section{Points clés}

- De rares cas du syndrome de fuite capillaire systémique (SFCS) après un vaccin contre le SRAS-CoV-2 à vecteur adénoviral ou à ARNm ont été recensés dans le monde. Certains sont survenus chez des patients ayant des antécédents de SFCS.

- Le SFCS est une maladie immunitaire potentiellement mortelle caractérisée par des épisodes d'hyperperméabilité endothéliale vasculaire transitoires et récurrents souvent déclenchés par une infection des voies respiratoires supérieures d'origine virale.

- L'hypotension, l'hémoconcentration, l'hypoalbuminémie et l'anasarque sont des symptômes caractéristiques du SFCS, en raison de la fuite importante de liquides et de protéines vers les tissus périphériques.

- Le SFCS aigu peut rapidement entraîner un choc et la défaillance de plusieurs organes. Son traitement vise surtout à corriger l'hypovolémie et à éviter les lésions aux organes vitaux; l'administration mensuelle prophylactique d'immunoglobulines peut prévenir la survenue de nouveaux épisodes.

- Les vaccins contre le SRAS-CoV-2 à vecteur adénoviral sont déconseillés aux personnes ayant des antécédents de SFCS.

Malgré la faible probabilité de processus infectieux, nous avons amorcé l'administration intraveineuse de liquides ainsi qu'une antibiothérapie empirique de pipéracilline/ tazobactam. Douze heures plus tard, le patient avait reçu plus de $6 \mathrm{~L}$ de liquides, mais sa pression artérielle était passée à 93/60 mm Hg et sa fréquence cardiaque, à 125 battements/min. La polyglobulie était toujours présente (taux d'hémoglobine de $223 \mathrm{~g} / \mathrm{L})$. Le patient a été hospitalisé à l'unité des soins intensifs (USI). Vu l'absence d'autres causes pouvant expliquer le choc, nous avons diagnostiqué un syndrome de fuite capillaire systémique (SFCS).

Dans les 24 heures suivant son admission, le patient a reçu plus de $10 \mathrm{~L}$ de liquides par voie intraveineuse; or, ses taux d'hémoglobine et d'acide lactique sont demeurés élevés (tableau 1), et son taux de créatinine a continué d'augmenter. 
Sa pression veineuse centrale était stable, oscillant entre 0 et $1 \mathrm{~mm} \mathrm{Hg}$. Il n'a pas eu besoin de vasopresseurs; seule une oxygénothérapie transitoire a été nécessaire. Le patient a développé une anasarque importante et pris $15 \mathrm{~kg}$. Son état hémodynamique a fini par s'améliorer, ses résultats de laboratoire sont redevenus normaux, et le patient a obtenu son congé 4 jours plus tard.

Diverses causes pouvant expliquer l'hypotension, la polyglobulie et l'hypoalbuminémie ont été envisagées, mais elles ont toutes été éliminées (tableau 2). Comme le SFCS est apparu 2 jours après la vaccination contre le SRAS-CoV-2 et qu'aucun autre déclencheur n'a été relevé, nous avons soupçonné qu'il s'agissait d'une réaction indésirable au vaccin ChAdOx1 nCOV-19, réaction que nous avons déclarée au bureau local de santé publique.

\section{Interprétation}

Le SFCS est un trouble rare associé à des épisodes récurrents d'extravasation de liquides et de protéines dans l'espace interstitiel $^{1,2}$. À ce jour, moins de 500 cas ont été signalés. Le SFCS peut être difficile à diagnostiquer parce que ses manifestations sont souvent précédées de prodromes pseudogrippaux et qu'on peut le confondre avec un sepsis. Il n'existe pas non plus de critères diagnostiques précis. Une fois toutes les autres causes de choc éliminées, la triade classique combinant hypotension, hémoconcentration et hypoalbuminémie vient appuyer le diagnostic de SFCS². Associées à un œdème généralisé, ces 3 caractéristiques témoignent de l'hyperperméabilité vasculaire et de l'hypovolémie extrême causées par ce syndrome.

Le processus physiopathologique exact du SFCS est en grande partie inconnu. Une exacerbation est généralement déclenchée par une infection des voies respiratoires supérieures d'origine virale ${ }^{1,3-5}$. La réponse immunitaire exagérée et la régulation positive des médiateurs inflammatoires et angiogéniques solubles observées durant une crise (poussée) semblent liées à l'hyperperméabilité endothéliale vasculaire ${ }^{2}$. Une gammapathie monoclonale à signification indéterminée (principalement de type IgG $\kappa$ ) est présente chez $68 \%-85 \%$ des patients atteints d'un SFCS, mais le rôle pathogénique des paraprotéines reste à déterminer². Des rapports de cas font état d'arrêts cardiaques déclenchés par l'influenza de type A chez des patients ayant un SFCS, ce qui n'est pas sans rappeler ce qu'a vécu notre patient en $2017^{5,6}$. Il est probable que l'arrêt cardiaque vécu à l'époque soit survenu au cours d'un SFCS non diagnostiqué malgré la présence d'une hypoalbuminémie ( $29 \mathrm{~g} / \mathrm{L}$ ), d'une hémoconcentration et d'une hypovolémie. Un SFCS n'a toutefois jamais été soupçonné avant la présente hospitalisation.

Les expériences quasi fatales de notre patient montrent bien qu'un SFCS non diagnostiqué peut être potentiellement mortel. On estime d'ailleurs que le taux de mortalité à 10 ans associé au SFCS est de $25 \%-34 \%{ }^{1,4}$. Outre le choc et les défaillances rénale et cardiopulmonaire attribuables à la baisse du volume intravasculaire, des thromboembolies et un syndrome des loges peuvent survenir. Le SFCS peut être de grade 1 (hypotension répondant à l'hydratation orale), de grade 2 (administration intraveineuse de liquides sans hospitalisation), de grade 3 (menace pour la vie et hospitalisation sur une USI nécessaire) ou de grade 4 (fatal) ${ }^{1}$.

Seule la réanimation liquidienne s'est avérée efficace pour freiner ou ralentir la progression d'une poussée de SFCS². La plupart des épisodes se résorbent d'eux-mêmes en 4 jours ${ }^{1}$. La fréquence de récurrence du SFCS varie, allant d'une fois par semaine à 1 fois tous les 10 ans. L'administration prophylactique mensuelle d'immunoglobulines par voie intraveineuse peut réduire cette fréquence ${ }^{1,2}$.

D'après l'Organisation mondiale de la Santé (OMS), 3,8 milliards de doses de vaccins contre le SRAS-CoV-2 ont été administrées

Tableau 1 : Résultats des analyses de laboratoire faites durant les 4 jours d'hospitalisation du patient

\begin{tabular}{|c|c|c|c|c|c|c|c|}
\hline Analyse de laboratoire & Admission & $4 \mathrm{~h}$ & $12 \mathrm{~h}$ & $24 \mathrm{~h}$ & $48 \mathrm{~h}$ & $72 \mathrm{~h}$ & $96 \mathrm{~h}$ \\
\hline Hémoglobine, g/L (plage normale 130-180 g/L) & 224 & 226 & 223 & 184 & 142 & 131 & 136 \\
\hline Hématocrite, \% (plage normale $39 \%-52 \%$ ) & 65 & 68,8 & 68 & 54,8 & 41,9 & 38,4 & 40 \\
\hline Leucocytes, $\times 10^{9} / \mathrm{L}$ (plage normale 4,4-11,0×109/L) & 14,5 & 19,7 & 24,5 & 21,5 & 11,4 & 7,6 & 6,9 \\
\hline Neutrophiles, $\times 10^{9} / \mathrm{L}$ (plage normale $1,8-7,0 \times 10^{9} / \mathrm{L}$ ) & 10,5 & 15,7 & 21,3 & 16,7 & 7,6 & 4,6 & 4,3 \\
\hline Lymphocytes, $\times 10^{9} / \mathrm{L}$ (plage normale $1,0-4,0 \times 10^{9} / \mathrm{L}$ ) & 2,4 & 2,2 & 2,4 & 3,2 & 2,5 & 2,1 & 2 \\
\hline Plaquettes, $\times 10^{9} / \mathrm{L}$ (plage normale $140-440 \times 10^{9} / \mathrm{L}$ ) & 222 & 237 & 251 & 202 & 173 & 164 & 174 \\
\hline Albumine, $\mathrm{g} / \mathrm{L}$ (plage normale $34-55 \mathrm{~g} / \mathrm{L}$ ) & 28 & N. R. & N. R. & 25 & N. R. & N. R. & 38 \\
\hline Créatinine, $\mu \mathrm{mol} / \mathrm{L}$ (plage normale $62-115 \mu \mathrm{mol} / \mathrm{L}$ ) & 133 & 133 & 159 & 122 & 90 & 71 & 79 \\
\hline Lactate, $\mathrm{mmol} / \mathrm{L}$ (plage normale $0,5-2,2 \mathrm{mmol} / \mathrm{L}$ ) & N. R. & N. R. & 3,8 & 3,3 & 1,1 & N. R. & N. R. \\
\hline D-dimères, $\mu \mathrm{g} / \mathrm{L}$ (plage normale $0-500 \mu \mathrm{g} / \mathrm{L}$ ) & 353 & N. R. & N. R. & N. R. & N. R. & N. R. & N. R. \\
\hline Temps de prothrombine, RIN (plage normale $0,9-1,1$ ) & 1,1 & N. R. & N. R. & N. R. & N. R. & N. R. & N. R. \\
\hline Temps de céphaline activée, s (plage normale 20,1-26,4 s) & 28,4 & N. R. & N.R. & N. R. & N. R. & N. R. & N. R. \\
\hline
\end{tabular}


Tableau 2 (partie 1 de 2) : Causes possibles de polyglobulie, d'hypoalbuminémie et d'hypotension, et raisons de leur exclusion chez le patient

Trouble Diagnostic différentiel

\section{Raisons d'exclusion}

\section{Polyglobulie}

Polyglobulie primaire

Polyglobulie secondaire

Polyglobulie relative

Hypoalbuminémie

Hypotension et choc

- Absence de mutation du gène $B C R-A B L t(9 ; 22)$ d'apnée selon le score STOP-BANG

- Aucune maladie pulmonaire chronique connue

- Pas d'antécédents de séjour en haute altitude

- Taux d'érythropoïétine non élevé

- Non exclu (plage normale $<0,15 \mathrm{~g}$ protéine/g créatinine) (bilan lipidique normal à jeun) ou à une cirrhose
Innée :

Malformation cardiaque congénitale

Acquise :

Érythrémie

Leucémie

Hypoxie chronique ou anomalies cardiopulmonaires

Tumeurs sécrétant de l'érythropoïétine

Déshydratation Syndrome de fuite capillaire

Syndrome néphrotique

Mauvaise alimentation ou cirrhose du foie

Sepsis

Choc cardiogénique ou obstructif
- Résultats normaux à l'échocardiogramme transthoracique

- Absence de mutation (Val671Phe) du gène Janus kinase 2 (JAK2)

- Aucun symptôme clinique d'apnée obstructive du sommeil et faible risque de ce type

- Pas de syndrome obésité-hypoventilation et pas d'hypercapnie diurne

- Taux d'érythropoiétine non élevé : 4,6 UI/L (plage normale 5,0-16,0 UI/L)

- Taux d'hémoglobine et d'hématocrite normaux entre les épisodes aigus

- Pas de protéinurie, rapport protéine/créatinine urinaire négatif : indétectable

- Pas de signe d'hyperlipidémie pouvant être liée à un syndrome néphrotique

- Correction rapide du taux d'albumine sérique ne concordant pas avec une hypoalbuminémie attribuable à une mauvaise alimentation

- Aucun signe caractéristique de cirrhose du foie ou d'ascite à la TDM abdominale

- Taux normaux de procalcitonine $(0,28 \mu \mathrm{g} / \mathrm{L}$ [plage normale $0,00-0,39 \mu \mathrm{g} / \mathrm{L}$ ]) et de protéine C-réactive $(4,9 \mathrm{mg} / \mathrm{L}$ [plage normale $0,0-10,0 \mathrm{mg} / \mathrm{L}$ ])

- Aucun signe d'infection à la radiographie thoracique et à la TDM abdominale

- Deux résultats d'hémoculture négatifs

- Résultat négatif à la culture urinaire

- Résultat négatif au dépistage (RT-PCR) du SRAS-CoV-2 (trousse de diagnostic direct de la COVID-19)

- Résultat négatif au dépistage sérologique du SRAS-CoV-2 (IgG)

- Résultat négatif au dépistage de virus et de pathogènes respiratoires courants, du virus respiratoire syncytial, de l'influenza de type $\mathrm{A}$ ( $\mathrm{H} 1$ et $\mathrm{H} 3$ ); de l'influenza de type $\mathrm{B}$; des virus parainfluenza de types 1, 2, 3 et 4; du métapneumovirus humain; d'entérovirus et de rhinovirus; des coronavirus NL63, HKU1, OC43 et 229E; d'adénovirus; de Bordetella pertussis et parapertussis; de Chlamydophilia pneumoniae; et de Mycoplasma pneumoniae

- Résultat négatif au dépistage sérologique des VIH-1 et 2 (test combiné faisant appel au p24)

- Résultat normal à l'échographie ciblée en traumatologie (FAST, pour focussed assessment with sonography for trauma) faite au service des urgences

- Résultat normal à l'électrocardiogramme

- Résultat normal à l'échocardiographie transthoracique et résultat négatif à une épreuve d'effort récemment réalisée en consultation externe

- Absence d'œdème pulmonaire et de pression veineuse centrale élevée

- Taux normaux d'enzymes cardiaques (troponine $<3 \mathrm{ng} / \mathrm{L}$ [plage normale $0-18 \mathrm{ng} / \mathrm{L}$ ]) et de D-dimères $(353 \mu \mathrm{g} / \mathrm{L}$ [plage normale $<500 \mu \mathrm{g} / \mathrm{L}$ ] $)$

- Résultat négatif à la scintigraphie V/Q 
Tableau 2 (partie 2 de 2) : Causes possibles de polyglobulie, d'hypoalbuminémie et d'hypotension, et raisons de leur exclusion chez le patient

Anaphylaxie

Edème de Quincke héréditaire

\section{Autres maladies} exclues

Réaction à un
- Absence de signes ou de symptômes cliniques normalement associés à l'anaphylaxie (p. ex., atteinte respiratoire, érythème, prurit, œdème de Quincke et symptômes gastrointestinaux persistants)

- Taux normal de tryptase : 3,0 $\mu \mathrm{g} / \mathrm{L}$ (plage normale $<11 \mu \mathrm{g} / \mathrm{L}$ )

Activité normale de la C4 estérase $(0,20 \mathrm{~g} / \mathrm{L}$ [plage normale $0,13-0,40 \mathrm{~g} / \mathrm{L}$ ]) et de la C1 estérase $(0,97 \mathrm{~g} / \mathrm{L}$ [plage normale $0,69-1,42 \mathrm{~g} / \mathrm{L}$ ]) médicament Absence de facteurs épidémiologiques habituellement associés à ces diagnostics

anticancéreux

Lymphohistiocytose

hémophagocytaire

Fièvre hémorragique

virale

Empoisonnement à

la suite d'une morsure

de serpent

Remarque : IgG = immunoglobulines G, RT-PCR = test d'amplification en chaîne par polymérase couplé à une transcription inverse, TDM = tomodensitométrie.

dans le monde (en date du 29 juillet 2021; tableau de bord de l'OMS sur la COVID-19 accessible en anglais au https://covid19.who.int). Les effets secondaires du vaccin sont généralement légers et localisés, quoique de rares réactions indésirables graves peuvent survenir (péricardite, myocardite, anaphylaxie, syndrome de GuillainBarré ou thromboembolies s'accompagnant d'un faible taux de plaquettes, surtout après un vaccin à vecteur adénoviral) ${ }^{7}$.

En avril 2021, l'Agence européenne des médicaments (EMA) a signalé 6 cas de SFCS (dont 1 décès) suivant l'administration du vaccin ChAdOx1 nCOV-198. Trois des patients avaient des antécédents de SFCS. Plus de 78 millions de doses de ce vaccin ont été administrées en Europe, où le taux rapporté de SFCS est de 1 cas par 13 millions de doses ${ }^{8}$. En juin 2021, Santé Canada a signalé le premier cas de SFCS au pays chez 1 personne ayant reçu le vaccin ChAdOx1 nCOV-199. L'Agence de réglementation des médicaments et des produits de santé (MHRA) du Royaume-Uni a fait état de 8 possibles cas de SFCS survenus peu après l'administration de ce vaccin 7 . Notre patient a obtenu un score d'au moins 4 sur l'échelle Naranjo de probabilité de réaction médicamenteuse indésirable $\mathrm{e}^{10}$; il est donc possible que le vaccin ChAdOx1 nCOV-19 ait exacerbé le SFCS.

De nombreux organismes sanitaires ont conclu que bien qu'il soit rare, le SFCS devrait être considéré comme une réaction indésirable grave et potentiellement mortelle au vaccin ChAdOx1 nCOV-19. Ce dernier est aujourd'hui déconseillé aux patients ayant reçu un diagnostic de $\mathrm{SFCS}^{7-9}$. Une recommandation semblable a été faite pour le vaccin Ad26.COV2.S (vaccin à vecteur adénoviral de Johnson \& Johnson-Janssen), après que l'EMA ait signalé en juillet 20213 cas de SFCS grave, dont 2 décès, potentiellement liés à ce vaccin ${ }^{8}$.

En juin 2021, une étude de série de cas a indiqué que 3 personnes ont consulté pour un SFCS aigu dans les 2 jours suivant la réception d'un vaccin contre le SRAS-CoV- $2^{11}$. Elles présentaient toutes une gammapathie monoclonale à signification indéterminée et des antécédents de SFCS. Ces 3 personnes avaient reçu des vaccins différents : Ad26.COV2.S, ARNm-1273 (Moderna) et BNT162b2 (Pfizer-BioNTech). À notre connaissance, aucun autre cas de SFCS lié aux vaccins contre le SRAS-CoV-2 à ARNm n'a été signalé, et aucune mise en garde ni recommandation n'a été faite en ce qui a trait au SFCS et aux vaccins ARNm-1273 et BNT162b2. Il reste à déterminer si les exacerbations déclarées ont été provoquées par les vaccins à vecteur adénoviral eux-mêmes ou par l'antigène (protéine de spicule) du SRAS-CoV-2.

Les vaccins à ARNm peuvent tout de même être utilisés chez les patients ayant des antécédents de SFCS si les bienfaits en surpassent les risques. Après la vaccination, les médecins et les patients doivent faire preuve d'une grande vigilance; en effet, une exacerbation peut survenir rapidement et une évaluation urgente pourrait être nécessaire. À notre avis, les patients devraient faire l'objet d'un suivi étroit pendant 7-10 jours après la vaccination. Ce suivi devrait comprendre une surveillance à domicile de la pression artérielle, de la fréquence cardiaque, du poids et de la diurèse, ainsi qu'une ou 2 mesures des taux d'hémoglobine et d'albumine. Nous recommandons également l'administration prophylactique d'immunoglobulines par voie intraveineuse avant la vaccination, si une administration mensuelle n'a pas déjà lieu.

\section{Conclusion}

Il s'agissait ici d'un cas d'exacerbation aiguë du SFCS après l'administration du vaccin ChAdOx1 nCOV-19. Le diagnostic et le traitement rapides du SFCS aigu sont cruciaux. Il faut envisager ce syndrome chez les patients qui viennent consulter et qui présentent un œdème généralisé, un malaise, une hypotension, une hémoconcentration et une hypoalbuminémie peu après avoir reçu un vaccin contre le SRAS-CoV-2, quel qu'il soit. Les antécédents de SFCS constituent aujourd'hui une contre-indication aux vaccins contre le SRAS-CoV-2 à vecteur adénoviral. Les professionnels de la santé devraient signaler tout soupçon de réaction indésirable aux vaccins contre le SRAS-CoV-2 à leur agence de santé publique. 


\section{Références}

1. Druey KM, Greipp PR. Narrative review: the systemic capillary leak syndrome. Ann Intern Med 2010;153:90-8.

2. Druey KM, Parikh SM. Idiopathic systemic capillary leak syndrome (Clarkson disease). J Allergy Clin Immunol 2017;140:663-70.

3. Sousa A, Len O, Escola-Verge L, et al. Influenza A virus infection is associated with systemic capillary leak syndrome: case report and systematic review of the literature. Antivir Ther 2016;21:181-3.

4. Gousseff M, Arnaud L, Lambert M, et al. The systemic capillary leak syndrome: a case series of 28 patients from a European registry. Ann Intern Med 2011;154: 464-71.

5. Ebdrup L, Druey KM, Druey K, et al. Severe capillary leak syndrome with cardiac arrest triggered by influenza virus infection. BMJ Case Rep 2018 Aug. 29; 2018:bcr2018226108. doi: 10.1136/bcr-2018-226108.

6. Lawrence JL, Hindi H. Capillary leak syndrome aggravated by influenza type A infection. Cureus 2018;10:e2554

7. Coronavirus vaccine - weekly summary of Yellow Card reporting. London (UK): Medicines \& Healthcare products Regulatory Agency (MHRA); 2021. Accessible ici : https://assets.publishing.service.gov.uk/government/uploads/system/ uploads/ attachment_data/file/1005194/Coronavirus_vaccine_-_summary_of_ Yellow_Card_reporting_14.07.21_clean.pdf (consulté le 18 juill. 2021).

8. Pharmacovigilance Risk Assessment Committee (PRAC). COVID-19 pandemic. Amsterdam (Netherlands): European Medicines Agency (EMA). Accessible ici : https://www.ema.europa.eu/ (consulté le 11 juin 2021).

9. Recalls and safety alerts: Health Canada issues label change on the AstraZeneca and COVISHIELD COVID-19 vaccines. Ottawa: Health Canada; 2021 June 29. Accessible ici : https://healthycanadians.gc.ca/recall-alert-rappel-avis/hc-sc /2021/75389a-eng.php (consulté le 28 juill. 2021).

10. Naranjo CA, Busto U, Sellers EM, et al. A method for estimating the probability of adverse drug reactions. Clin Pharmacol Ther 1981;30:239-45.

11. Matheny M, Maleque N, Channell N, et al. Severe exacerbations of systemic capillary leak syndrome after COVID-19 vaccination: a case series. Ann Intern Med 2021 Jun 15;L21-0250 [cyberpublication avant impression]. doi: 10.7326/L21-0250.

Intérêts concurrents : Aucun déclaré.

Cet article a été révisé par des pairs.

Les auteures ont obtenu le consentement du patient.

Affiliations : Départements de soins intensifs et de médecine interne (Robichaud), de pharmacie (C. Côté) et de médecine interne (F. Côté), CIUSSS du Saguenay-Lac-Saint-Jean, Chicoutimi, Qc.

Contributeurs : Toutes les auteures ont contribué à l'analyse des données et participé à parts égales à la rédaction du manuscrit; elles ont donné leur approbation finale pour la version destinée à être publiée et assument l'entière responsabilité de tous les aspects du travail.

Propriété intellectuelle du contenu : Il s'agit d'un article en libre accès distribué conformément aux modalités de la licence Creative Commons Attributions (CC BY-NC-ND 4.0), qui permet l'utilisation, la diffusion et la reproduction dans tout médium à la condition que la publication originale soit adéquatement citée, que l'utilisation se fasse à des fins non commerciales (c.-à-d. recherche ou éducation) et qu'aucune modification ni adaptation n'y soit apportée. Voir : https://creativecommons.org/licenses/by-nc-nd/4.0/deed.fr.

Correspondance : Julie Robichaud,

julie.robichaud.med@ssss.gouv.qc.ca 